\title{
Dynamic variations in epithelial-to-mesenchymal transition (EMT), ATM, and SLFN11 govern response to PARP inhibitors and cisplatin in small cell lung cancer
}

\begin{abstract}
C. Allison Stewart ${ }^{1}$, Pan Tong ${ }^{2}$, Robert J. Cardnell ${ }^{1}$, Triparna Sen ${ }^{1}$, Lerong Li ${ }^{2}$, Carl M. Gay ${ }^{1}$, Fatemah Masrorpour ${ }^{1}$, You Fan ${ }^{1}$, Rasha O. Bara ${ }^{1}$, Ying Feng ${ }^{3}$, Yuanbin Ru ${ }^{3}$, Junya Fujimoto ${ }^{4}$, Samrat T. Kundu ${ }^{1}$, Leonard E. Post ${ }^{3}$, Karen Yu $^{3}$, Yuqiao Shen ${ }^{3}$, Bonnie S. Glisson ${ }^{1}$, Ignacio Wistuba ${ }^{4}$, John V. Heymach ${ }^{1}$, Don L. Gibbons ${ }^{1}$, Jing Wang $^{2}$ and Lauren Averett Byers ${ }^{1}$

${ }^{1}$ Department of Thoracic Head \& Neck Medical Oncology, The University of Texas MD Anderson Cancer Center, Houston, TX 77030, USA

${ }^{2}$ Department of Bioinformatics and Computational Biology, The University of Texas MD Anderson Cancer Center, Houston, TX 77030, USA

${ }^{3}$ BioMarin Pharmaceutical, San Rafael, CA 94901, USA

${ }^{4}$ Department of Translational Molecular Pathology, The University of Texas MD Anderson Cancer Center, Houston, TX 77030, USA

Correspondence to: Lauren Averett Byers, email: Ibyers@mdanderson.org

Keywords: SCLC, SLFNI 1, ATM, EMT, PARP inhibitor

Received: December 10, $2016 \quad$ Accepted: January 19, $2017 \quad$ Published: February 15, 2017

Copyright: Stewart et al. This is an open-access article distributed under the terms of the Creative Commons Attribution License (CC-BY), which permits unrestricted use, distribution, and reproduction in any medium, provided the original author and source are credited.
\end{abstract}

\section{ABSTRACT}

Small cell lung cancer (SCLC) is one of the most aggressive forms of cancer, with a 5-year survival $<7 \%$. A major barrier to progress is the absence of predictive biomarkers for chemotherapy and novel targeted agents such as PARP inhibitors. Using a high-throughput, integrated proteomic, transcriptomic, and genomic analysis of SCLC patient-derived xenografts (PDXs) and profiled cell lines, we identified biomarkers of drug sensitivity and determined their prevalence in patient tumors. In contrast to breast and ovarian cancer, PARP inhibitor response was not associated with mutations in homologous recombination (HR) genes (e.g., BRCA1/2) or HRD scores. Instead, we found several proteomic markers that predicted PDX response, including high levels of SLFN11 and E-cadherin and low ATM. SLFN11 and E-cadherin were also significantly associated with in vitro sensitivity to cisplatin and topoisomerase1/2 inhibitors (all commonly used in SCLC). Treatment with cisplatin or PARP inhibitors downregulated SLFN11 and E-cadherin, possibly explaining the rapid development of therapeutic resistance in SCLC. Supporting their functional role, silencing SLFN11 reduced in vitro sensitivity and drug-induced DNA damage; whereas ATM knockdown or pharmacologic inhibition enhanced sensitivity. Notably, SCLC with mesenchymal phenotypes (i.e., loss of E-cadherin and high epithelial-to-mesenchymal transition (EMT) signature scores) displayed striking alterations in expression of miR200 family and key SCLC genes (e.g., NEUROD1, ASCL1, ALDH1A1, MYCL1). Thus, SLFN11, EMT, and ATM mediate therapeutic response in SCLC and warrant further clinical investigation as predictive biomarkers.

\section{INTRODUCTION}

In patients with small cell lung cancer (SCLC), responses to frontline chemotherapy are rapidly overcome by drug resistance [1]. We previously demonstrated that PARP1 is a promising novel therapeutic target in SCLC $[2,3]$ and multiple PARP inhibitor clinical trials have now been initiated. However, unlike other forms of 
lung cancer, there are currently no biomarkers to predict treatment response in SCLC. In other cancers, response to PARP inhibitors has been associated with defects in DNA damage response (DDR) (e.g., mutations in BRCA1/2, $A T M)$ [4, 5]. However, only $\sim 12 \%$ of SCLC tumors have mutations in DDR genes [6-8] and most of those mutations do not have a known or predicted deleterious effect on function [6].

One emerging biomarker for PARP inhibitors and chemotherapy in several cancers is Schlafen 11 (SLFN11) [9-11]. We and others have recently described an association between SLFN11 expression and sensitivity to various PARP inhibitors, including talazoparib and olaparib, in SCLC models [12-14]. However, some models with low levels of SLFN11 respond to PARP inhibition, whereas others with relatively high levels are resistant $[12,13]$. These findings suggest that SLFN11 is unlikely to be the only determinant of drug response in SCLC.

To characterize SCLC-specific biomarkers of therapeutic vulnerability, we performed a high-throughput, integrated proteomic, transcriptomic, and genomic analysis using SCLC PDX models, cell lines, and archival tumor specimens. We found that while DDR mutations and HR deficiency (HRD) scores were not predictive in SCLC, expression levels of several markers including SLFN11, ATM, and E-cadherin (reflecting epithelialto-mesenchymal transition (EMT) status) determined response to both PARP inhibitors and several classes of chemotherapy in preclinical models.

\section{RESULTS}

\section{Identification of biomarkers in preclinical SCLC models}

PDX models are derived from direct implantation of patient tumor biopsies into immunodeficient mice. PDX models retain both genetic similarities and drug response to the patient tumor from which they were developed and are useful for studying both tumor biology and preclinical testing $[15,16]$. SCLC PDX models were treated with vehicle or talazoparib (PARP inhibitor) and assigned to response groups, partial response (PR), stable disease (SD), or progressive disease (PD) based on tumor growth and percent change from baseline (Figure 1A; Supplementary Figure 1). Tumors from untreated (vehicle) PDXs were analyzed by reverse-phase protein array (RPPA) and RNA sequencing (RNAseq) to identify pre-treatment protein and mRNA differences between those that were sensitive (PR or SD) or resistant (PD) to single-agent talazoparib. Of 170 proteins and/ or phosphorylated proteins quantified by RPPA, low $\operatorname{ATM}(\mathrm{FC}=-2.32, \mathrm{P}=0.009)$ and high SLFN11 $(\mathrm{FC}=5.11$, $\mathrm{P}=0.014)$ protein expression were the most significantly associated with talazoparib response in the PDX models (Figure 1B). High CHK1 ( $\mathrm{FC}=-1.48, \mathrm{P}=0.017)$, IGF1R beta $(\mathrm{FC}=-1.73, \mathrm{P}=0.045)$, and $\mathrm{IRS} 1(\mathrm{FC}=-1.39, \mathrm{P}=0.025)$ protein levels were associated with resistance (Figure 1C). Protein biomarker results were further validated at the mRNA level which also showed an association between talazoparib response and high SLFN11 ( $\mathrm{FC}=38.82$, $\mathrm{P}=0.031)$, low ATM ( $\mathrm{FC}=-2.12, \mathrm{P}=0.004)$, and low CHEK1 $(\mathrm{FC}=-1.86, \mathrm{P}=0.003)$ expression in $\mathrm{PDX}$ models (Figure 1D). Interestingly, although the two PDXs with the lowest ATM levels both had partial responses to talazoparib, only one of these expressed high levels of SLFN11 (Supplementary Figure 2C). This suggests that either low ATM or high SLFN11 is sufficient to predict sensitivity to talazoparib and therefore that more than one biomarker may predict SCLC response to PARP inhibition.

After SLFN11, E-cadherin (a marker commonly associated with EMT status) was the next strongest protein marker correlated with sensitivity $(\mathrm{FC}=2.24$, $\mathrm{P}=0.158)$, although this did not reach statistical significance possibly due to the small sample size. Given previous findings by our group and others that EMT mediates resistance to several targeted therapies in nonsmall cell lung cancer (NSCLC), we further investigated the association between E-cadherin and talazoparib sensitivity using an EMT gene expression signature [17]. Consistent with the E-cadherin protein data, we found that PDX models that were resistant to talazoparib (PD) were more mesenchymal than those with PR or SD (mean difference $=-2.21, \mathrm{P}=0.052$ ) (Figure 1E).

Finally, we performed immunohistochemistry (IHC) on tumors from the vehicle-treated PDXs for the top two biomarkers (SLFN11 and ATM) to independently validate the RPPA results (Figure 1F). H-scores for the ATM and SLFN11 levels were highly correlated with RPPA measurements (rho $=0.62[\mathrm{P}=0.02]$ and $0.82[\mathrm{P}=0.0006]$, respectively; Supplementary Figure 2D). As expected, SLFN11 H score was also correlated with PDX sensitivity ( $\mathrm{FC}=8.639, \mathrm{P}=0.03)$, whereas ATM H score was borderline significant $(\mathrm{FC}=-3.446, \mathrm{P}=0.09$; Figure $1 \mathrm{~F})$. This may have been due to the semi-quantitative nature of IHC compared with RPPA, which has greater sensitivity at lower protein expression levels [18]. These results suggest that IHC for SLFN11 and ATM in patient biopsy specimens could be a viable method for identifying patients with a higher likelihood of response to PARP inhibition.

\section{Biomarkers from other cancer types do not predict PARP inhibitor response in SCLC}

In other cancers such as ovarian and breast, mutations in HR genes (e.g., BRCA1/2, ATM) and/ or elevated HRD scores (e.g., Myriad Genetics testing scores) [19] predict patient response to PARP inhibitors. We found no association between $\mathrm{PDX}$ in vivo sensitivity to talazoparib and the HRD score $(\mathrm{FC}=1.081, \mathrm{P}=0.748$; Figure $1 \mathrm{G})$. Consistent with this, HRD score also was not correlated with in vitro response to cisplatin ( $\mathrm{rho}=-0.136$, $\mathrm{P}=0.674)$, talazoparib ( $\mathrm{rho}=0.175, \mathrm{P}=0.586$ ) or olaparib 
(rho=0.290, $\mathrm{P}=0.360$ ) (Supplementary Figure 2A) in a panel of 12 cell lines. We next tested whether the specific mutations were predictive in SCLC using a commercially available next-generation sequencing platform that includes targeted sequencing of 315 cancer-related genes plus select introns from 28 genes often rearranged or altered in solid tumor cancers (FoundationOne). PDX response did not correlate with the number of nongermline mutations $(\mathrm{FC}=-1.514, \mathrm{P}=0.083$; as determined by SGZ algorithm) (Figure $1 \mathrm{G}$ ) or overall mutation burden $(\mathrm{FC}=-1.347, \mathrm{P}=0.156$; Supplementary Figure 2B). Furthermore, we found loss or mutation of TP53 and/or RB1 (hallmarks of SCLC) in the vast majority of PDX tumors; however, the mutations observed in genes previously associated with PARP inhibitor sensitivity were not predicted to be functionally relevant, nor were they significantly associated with talazoparib response in vivo (Figure 1H). These findings suggest that the ability of HR gene mutations and/or HRD scores to predict response to PARP inhibitors is disease-specific and unlikely to be a reliable method for selecting SCLC patients for treatment. Overall, we identified a number of new markers of sensitivity and resistance to PARP inhibitors in PDX models of SCLC (Table 1).

\section{SLFN11 and E-cadherin are the top biomarkers of PARP inhibitor response in SCLC cell lines}

Using an approach similar to the PDX analysis, we then used a panel of 51 SCLC cell lines to validate markers associated with drug sensitivity. Baseline expression of 170 proteins, including SLFN11 and ATM, were quantified by
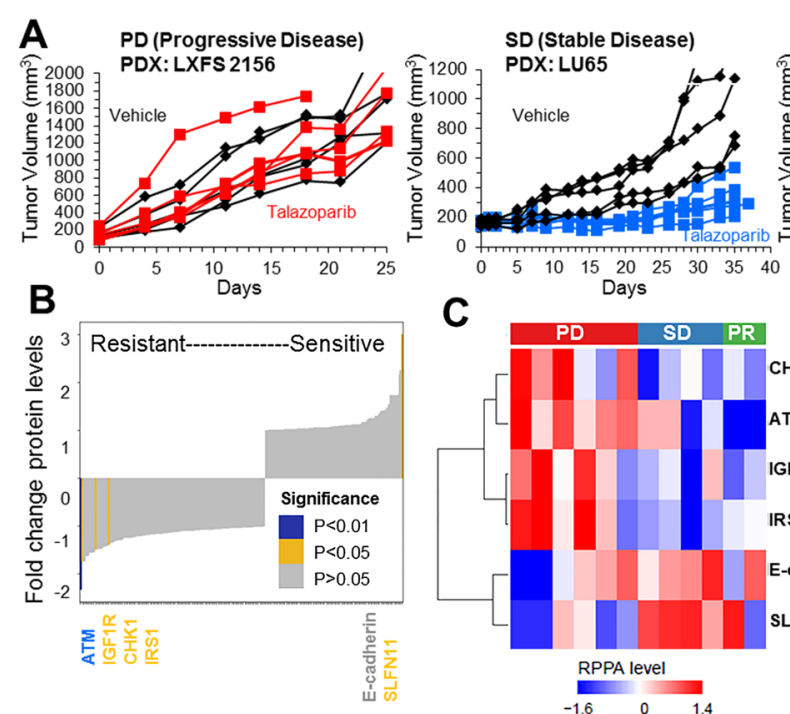

C
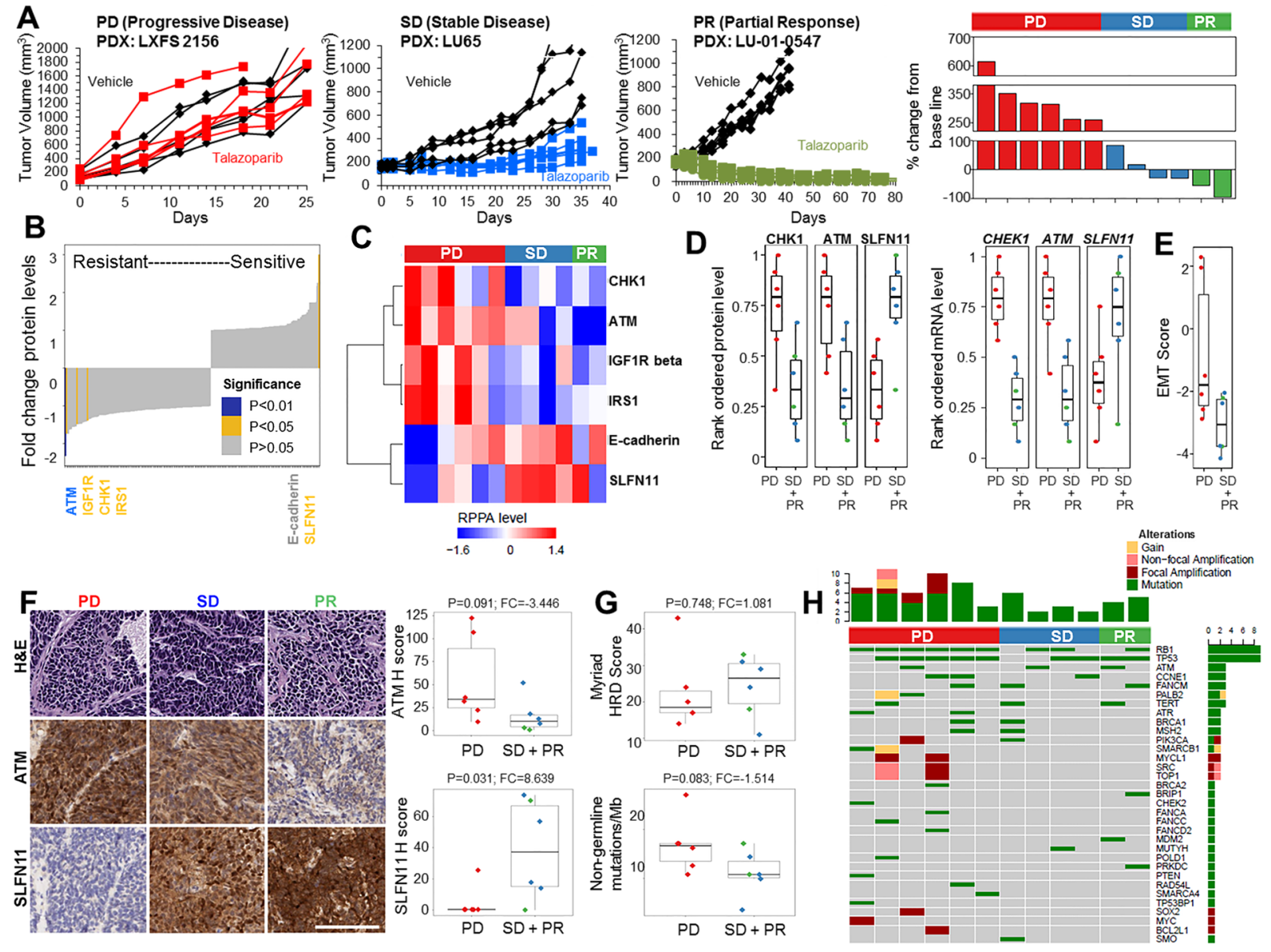

Figure 1: PDX Models with High SLFN11 and Low ATM Expression Levels Are More Sensitive to Talazoparib. A. Examples of PDX response to single-agent talazoparib and percent change in tumor volume from baseline for individual PDX models. B, and C. Regression analysis of 12 PDX models grouped as having progressive disease (PD, n=6), stable disease $(\mathrm{SD}, \mathrm{n}=4)$, or partial response $(\mathrm{PR}, \mathrm{n}=2)$ following treatment with single-agent talazoparib identifies high SLFN11 and low ATM expression and CHK1, IGF1R beta, and IRS1 as markers of sensitivity. D. Analysis of CHK1, ATM, and SLFN11 protein and mRNA expression across response groups. E. EMT score is correlated with PDX resistance to talazoparib. F. Immunolocalization of SLFN11 and ATM in PDX models from the 3 response groups. SLFN11, but not ATM H score, predicts PDX response. G. Myriad HRD score and FoundationOne non-germline mutational burden do not predict PDX response to talazoparib. H. Oncoprint representation of FoundationOne mutations identified in DDR genes or mutations previously shown to occur in SCLC. 
Table 1: Markers of talazoparib (PARP inhibitor) response in PDX models of SCLC

\begin{tabular}{lccc}
\hline & Markers of Talazoparib Response in vivo (PDX) & \\
\hline Mensitivity & Marker & P-value & Fold Change \\
\hline SLFN11 (protein) & 0.013 & 5.10 \\
SLFN11 (mRNA) & 0.031 & 38.82 \\
E-cadherin (protein) & 0.158 & 2.24 \\
CDH1 (mRNA) & 0.137 & 7.11 \\
EMT score & 0.05 & -2.24 (mean difference) \\
ATM (protein) & 0.009 & -2.32 \\
ATM (mRNA) & 0.004 & -2.12 \\
CHK1 (protein) & 0.017 & -1.48 \\
CHEK1 (mRNA) & 0.003 & -1.86 \\
IGF-1R (protein) & 0.044 & -1.73 \\
IRS1 (protein) & 0.025 & -1.39 \\
Myriad HRD & 0.748 & 1.081 \\
& FMI Non-germline mutations & 0.083 & -1.514 \\
FMI All mutations & 0.156 & -1.347 \\
PAR (ELISA) & 0.124 & 1.67 \\
& PARP1 (protein) & 0.614 & -1.06 \\
\hline
\end{tabular}

RPPA in untreated cell lines and correlated with sensitivity to PARP inhibitors (talazoparib, olaparib) and chemotherapy (cisplatin) (Figure 2A). As with the PDXs, for all 3 drugs, SLFN11 was the strongest marker of sensitivity by Spearman correlation (cisplatin, $\mathrm{P}<0.0001$; talazoparib, $\mathrm{P}<0.0001$; olaparib, $\mathrm{P}=0.02$ ). In addition, E-cadherin was again identified as a top marker of sensitivity to cisplatin $(\mathrm{P}<0.007)$, talazoparib $(\mathrm{P}<0.002)$, and olaparib $(\mathrm{P}=0.021)$. Statistical analysis of the association between ATM levels and drug response was limited by the fact that only 4 cell lines in the panel had low ATM levels.

\section{SLFN11 predicts response to multiple drug classes and immune marker expression}

SLFN11 protein and mRNA expression levels were highly concordant across the 51 SCLC cell lines $(\mathrm{Rho}=0.81, \mathrm{P}<0.0001$; Supplementary Figure 4A) and bimodally distributed (based on bimodal index $[\mathrm{BI}]=2.76$ and 3.01, respectively; Figure 2B and Supplementary Figure 4A). Using the BI to define cell lines with high versus low SLFN11 levels [20], we found that cell lines with high SLFN11 protein expression were significantly more sensitive to cisplatin $(\mathrm{FC}=-3.415, \mathrm{P}=0.0002)$ and talazoparib $(\mathrm{FC}=-4.367, \mathrm{P}=0.004)$ but not olaparib $(\mathrm{FC}=-$ 1.273, $\mathrm{P}=0.185$; Figure 2B). Similarly, the high SLFN11 mRNA expression group was also more sensitive to cisplatin $(\mathrm{FC}=-3.889, \mathrm{P}=0.04)$, talazoparib $(\mathrm{FC}=-4.664$, $\mathrm{P}=0.02)$, and olaparib $(\mathrm{FC}=-1.605, \mathrm{P}=0.04)$.

Cell lines with high endogenous SLFN11 levels (H209 and H526) were treated with cisplatin, olaparib, or talazoparib to determine whether drug treatment alters SLFN11 expression. Treatment with cisplatin and both PARP inhibitors reduced SLFN11 (Figure 2C) and E-cadherin (Supplementary Figure 7D) levels as measured by relative density of western blots. Furthermore, cisplatin treatment of lung adenocarcinoma cell lines with high endogenous levels of SLFN11 showed a subsequent reduction in expression (A549, $\mathrm{P}=0.002 ; \mathrm{H} 1944$, $\mathrm{P}<0.0001$; HCC $827, \mathrm{P}=0.001$ ), but not those with low levels (Calu6, $\mathrm{P}=0.785$; Figure $2 \mathrm{C}$ ). These data suggest SLFN11 levels in patient tumors may be similarly dynamic in response to treatment and, thus, biomarker analyses of initial diagnostic biopsies may be poor predictors of response to second or third line therapies.

Given the potential role of SLFN11 as a biomarker for several drug classes, we expanded our drug analyses of SCLC with high versus low SLFN11 expression levels to a larger screen of 526 oncology drugs in 63 SCLC cell lines [12]. We found that high SLFN11 protein levels, as determined by bimodal distribution, are associated with response to several drugs hitting the same targets or in common drug classes (Figure 2D and 2E). For example, high SLFN11 expression was correlated with sensitivity 
to alkylating agents $(n=14)$, TOP1 inhibitors $(n=6)$, TOP2A/B inhibitors $(n=7)$, DNA synthesis inhibitors $(n=3)$, and PARP inhibitors $(n=4)$. Interestingly, although high SLFN11 expression was not correlated with olaparib response in our analysis $(\mathrm{P}=0.185)$, it was correlated with response to the drug in the analysis of the larger cell line panel $(\mathrm{P}=0.002)$. Although SLFN11 has been associated with sensitivity to drugs in these classes previously $[9,12,13]$, ours is the most comprehensive list to date and includes additional drugs not previously reported.
In contrast to SLFN11, none of the other SLFN family members (SLFN5, SLFN12, SLFN13, and SLFN14) were associated with in vitro response to cisplatin or talazoparib (Supplementary Figure 3A and Supplementary Figure 3B).

As with the cell lines, we also observed a bimodal distribution of SLFN11 in publically available mRNA data from 70 early-stage, treatment-naïve SCLC patient tumors $(\mathrm{BI}=1.46$; Supplementary Figure 4A) [6], with $74 \%$ of tumors classified as SLFN11-high based on their BI. To better understand the functional role of SLFN11
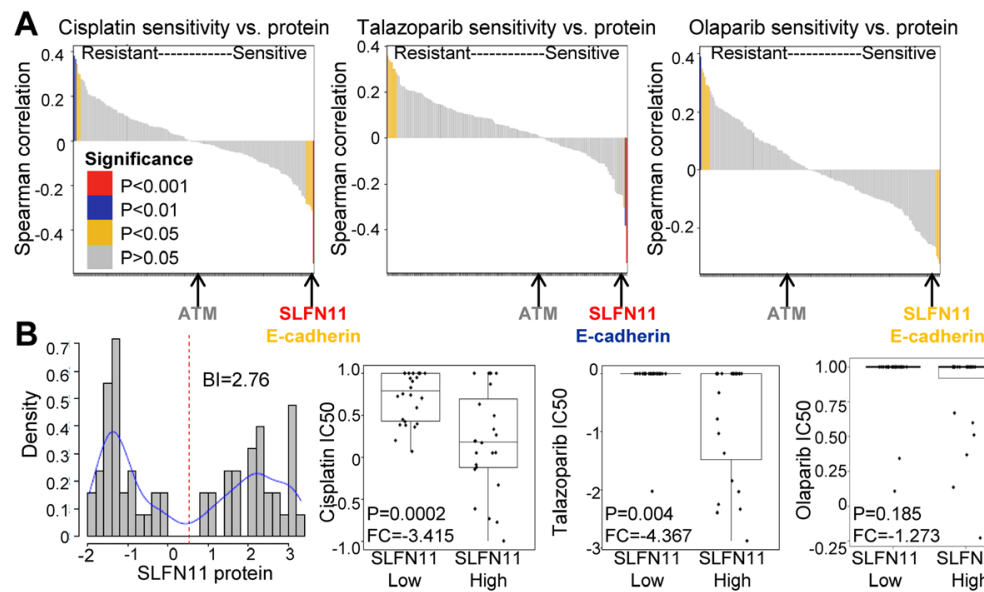

$\mathbf{F}$
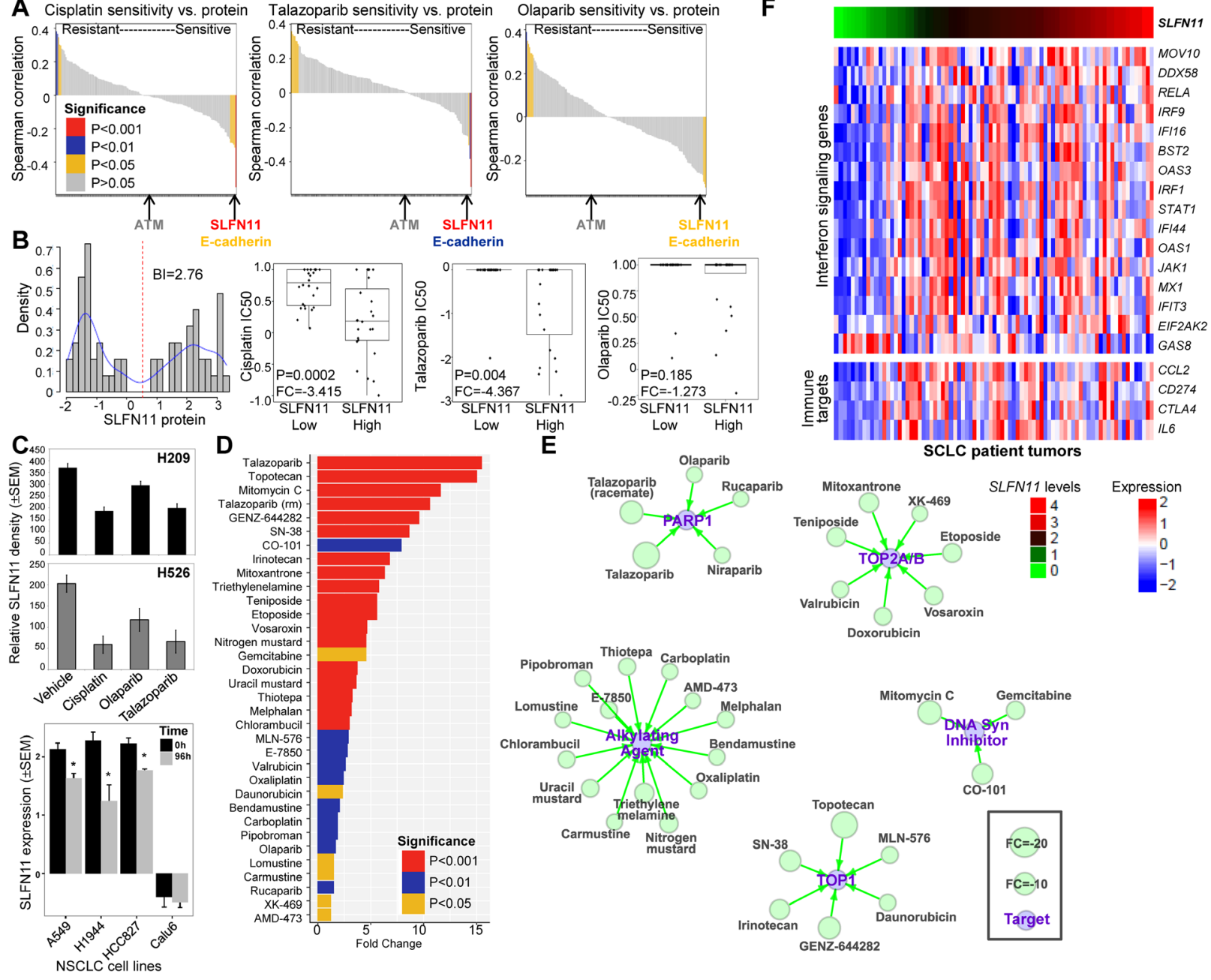

Figure 2: SLFN11 Protein Levels Are Correlated with Drug Sensitivity in SCLC. A. A correlation analysis of cisplatin, talazoparib, and olaparib sensitivity $\left(\mathrm{IC}_{50}\right.$ values) and 171 proteins in 51 SCLC cell lines shows that SLFN11 is the strongest predictor of sensitivity to both cisplatin and PARP inhibition. E-cadherin, but not ATM, is also correlated with drug sensitivity. B. SLFN11 expression is bimodal in SCLC cell lines, showing a switch-like pattern, and these naturally formed groups are correlated with drug sensitivity. Cell lines with higher SLFN11 protein expression levels have greater sensitivity to cisplatin and talazoparib but not olaparib. C. Treatment of H209 and H526 SCLC cell lines with $1 \mu \mathrm{M}$ cisplatin, $1 \mu \mathrm{M}$ olaparib, or $100 \mathrm{nM}$ talazoparib for $72 \mathrm{~h}$ reduced SLFN11 levels compared with vehicle-treated cells*, $\mathrm{P}<0.0005$. Treatment of A549, H1944, HCC827 NSCLC cell lines with $1 \mu$ M cisplatin for $96 \mathrm{~h}$ reduced SLFN11 levels, but not Calu6. *, P<0.002. D. Waterfall plot of drug sensitivity in SCLC. A comparison of high SLFN11 protein levels and the IC 50 values of 526 cancer drugs in 61 cell lines identified several drugs with similar targets, including PARP1 inhibitors, alkylating agents, TOP1 inhibitors, TOP2A/B inhibitors, and DNA synthesis inhibitors. E. A drug interaction network shows classes of oncology agents that are effective in SCLC cell lines with high SLFN11 levels. F. SLFN11-high SCLC has high expression levels of Type I IFN signaling molecules. IPA analysis identified IFN signaling as the top pathway associated with SLFN11 expression in SCLC patient tumors $\left(\mathrm{P}=6.6^{*} 10^{-6}\right)$.Heatmap of Type I IFN signaling genes and immunotherapy target genes ranked by association with SLFN11 levels in 70 tumors from treatmentnaïve SCLC patients $(\mathrm{FDR}=0.2)$. 
in tumors, we performed Ingenuity Pathway Analysis (www.ingenuity.com) comparing genes associated with high vs. low SLFN11 levels. Interestingly, these analyses revealed an enrichment of immune regulatory pathways, primarily interferon (IFN) signaling $\left(\mathrm{P}=6.6^{*} 10^{-6}\right)$, in SLFN11-high tumors. Consistent with this, we found that SLFN11 expression was positively correlated with Type I IFN pathway genes [21] in treatment-naïve SCLC patient tumors (Figure 2F). Furthermore, we screened a curated gene list enriched for immune targets [17] and found that high SLFN11 expression was positively correlated with PDL1 (CD274; $\mathrm{rho}=0.248, \mathrm{P}=0.025), \quad$ CCL2 (rho=0.271, $\mathrm{P}=0.014)$, CTLA4 (rho $=0.221, \mathrm{P}=0.046)$, and IL6 (rho $=0.226, \mathrm{P}=0.041$ ) in these same patient tumors (Figure 2F). This association was not observed in SCLC cell lines and stimulation of SCLC cells with a Type I IFN in vitro did not strongly modulate SLFN11 expression (Supplementary Figure 6A), suggests that immune cell expression of SLFN11 in patient tumors may be important.

\section{Knockdown of SLFN11 and ATM directly alters drug sensitivity}

Because of their roles in DNA repair [22, 23], we hypothesized that SLFN11 and ATM directly influence drug response in SCLC. H209 and DMS79 cell lines were selected for gene silencing based on their relatively high SLFN11 and ATM levels, and absence of ATM mutations. ATM mRNA and protein levels (Supplementary Figure 4C) were reduced by knockdown $(\mathrm{P}<0.0008$ and $<0.001$, respectively; Figures 3C, 3D, and Supplementary Figure 4C), as were SLFN11 mRNA and protein levels $(\mathrm{P}<0.0001$ and $<0.0004$, respectively; Figures 3A, 3B, and Supplementary Figure 4C). Differences in expression of ATM and SLFN11 were not present in control and scrambled samples for DMS79 or H209 (P>0.2). Silencing SLFN11 increased the resistance of the DMS79 (Figure 3A) and H209 (Figure 3B) cell lines to cisplatin $(\mathrm{P}<0.0005, \mathrm{P}=0.22)$, talazoparib $(\mathrm{P}<0.0001, \mathrm{P}<0.005)$ and olaparib $(\mathrm{P}<0.005, \mathrm{P}=0.119$; Supplementary Table 1). Conversely, silencing of ATM increased the sensitivity of DMS79 (Figure 3C) and H209 (Figure 3D) to cisplatin $(\mathrm{P}=0.20, \mathrm{P}<0.0001)$, talazoparib $(\mathrm{P}<0.009, \mathrm{P}<0.0001)$ and olaparib $(\mathrm{P}<0.006, \mathrm{P}<0.0001$; Supplementary Table 1). These findings demonstrate that both SLFN11 and ATM levels play a direct role in regulating cisplatin and PARP inhibitor sensitivity/resistance in SCLC.

Based on our findings, we hypothesized that a small molecule inhibitor of ATM may sensitize SLFN11low cell lines to PARP inhibition. Six SCLC cell lines (H82, H69, H841, H865, H2198, and H446) were treated with the ATM inhibitor KU55933 with or without the PARP inhibitors olaparib or talazoparib. The cell lines were not sensitive to treatment with the ATM inhibitor alone. However, in 5 of the 6 cell lines, the addition of the ATM inhibitor resulted in a modest sensitization to either of the PARP inhibitors. Furthermore, as predicted, the greatest sensitization was seen in cell lines with the lowest SLFN11 levels ( $\mathrm{P}<0.05$ for both PARP inhibitors; Figure 3E).

\section{High SLFN11 enhances DNA damage response}

We hypothesized that higher SLFN11 levels sensitize cells to DNA-damaging agents because of SLFN11's roles in sequestering RPA and disrupting checkpoint maintenance and HR repair [22]. To test this hypothesis, we looked at markers of DDR after SLFN11 knockdown with and without low doses of PARP inhibitor or cisplatin treatment. Low doses were used to minimize cytotoxicity, so as to analyze DNA damage rather than induction of apoptosis. We found that SLFN11 knockdown alone did not increase expression of $\gamma \mathrm{H} 2 \mathrm{AX}$ or change PARP1 levels (Figure 4A). However, the addition of treatment with PARP inhibitors or cisplatin for $24 \mathrm{~h}$ prevented the induction of DNA damage (as indicated by $\gamma \mathrm{H} 2 \mathrm{AX}$ expression) (Figure 4A), suggesting that SLFN11 plays a direct role in cellular accumulation of DNA damage following stimulation.

\section{Function and regulation of SLFN11 in SCLC}

Silencing of SLFN11 or ATM did not alter PARP1 levels (Figure 4B), and PARP1 itself did not predict sensitivity to PARP inhibitors in our SCLC PDXs or cell lines. One mechanism of PARP inhibitor resistance that has been described for other cancer types is the downregulation of PARP1 itself [24], which we hypothesized directly affects SLFN11 levels. Thus, we generated cell lines with stable knockdown of PARP1 with shRNA and assessed their SLFN11 levels. As shown by densitometric analysis of western blots, the knockdown cell lines had less SLFN11 (Figure 4B), regardless of the degree of PARP1 reduction. This suggests that decreased PARP1, as seen in some models of acquired resistance to PARP inhibitors, may play a role in regulating SLFN11 although further investigation is needed.

In other cancers, including Ewing sarcoma, SLFN11 has been described as an ETS transcription factor response gene [25]. Thus, we tested whether similar regulation of SLFN11 occurs in SCLC. Expression of 26 ETS transcription factors was correlated with SLFN11 and PARP1 mRNA levels and with drug sensitivity (Supplementary Table 2). Of these, only EHF was correlated with SLFN11 ( $\mathrm{P}=0.048$ in cell lines, $\mathrm{p}=0.004$ in tumors; Supplementary Figure $6 \mathrm{~B}$ ) and with in vitro drug sensitivity $(\mathrm{P}=0.045$; Supplementary Figure 6C). Like SLFN11, EHF was elevated in SCLC tumors (versus normal adjacent tissues) (Supplementary Table 3) and bimodally expressed $(\mathrm{BI}=2.66$ in cell lines, 1.61 in tumors) [26]. Suppression of EHF levels reduced SLFN11 expression (DMS79, $\mathrm{P}=0.003$; Figure 4C). Overall, this 
suggests that the ETS transcription factors regulating SLFN11 may be cancer-specific.

SLFN11 levels are sensitive to hypermethylation of the SLFN11 promoter [27]. To determine whether epigenetic methylation also plays a role in SLFN11 levels in SCLC, we analyzed 35 SCLC cell lines by Infinium HumanMethylation450 BeadChip array (Illumina). Levels of methylation in $19 \mathrm{CpG}$ regions were linked to SLFN11. We identified 3 regions within the TSS that were negatively correlated with SLFN11 mRNA expression and 2 of these were also correlated with resistance to cisplatin, talazoparib, and olaparib (Supplementary Figure 8A-8D). The cg18608369 region, which has been reported to be correlated with cisplatin and carboplatin resistance in the NCI60 cell line panel [27], was not correlated with cisplatin resistance in SCLC $(\mathrm{P}=0.45)$. Treatment with a demethylase and/or HDAC inhibitor did not increase SLFN11 expression, and demethylation with decitabine did not improve SCLC cell lines' sensitivity to cisplatin or PARP inhibitors (data not shown). Although SLFN11 promoter methylation is associated with cisplatin and PARP inhibitor resistance, demethylation and/or HDAC inhibition were not sufficient to upregulate SLFN11 levels (Supplementary Figure 8E). MGMT methylation was not strongly correlated with SLFN11 expression or sensitivity to talazoparib or olaparib (data not shown).

\section{E-cadherin expression predicts drug sensitivity in SCLC}

E-cadherin was a top marker of drug sensitivity in PDX models and cell lines (Figures 1B, 1C, and 2A). Therefore, to further establish whether the differences in E-cadherin reflected a broader EMT program, we investigated miRNAs and genes with established roles in EMT (e.g., miR200 family members, ZEB1, EMT gene signatures). Specimens were defined as mesenchymal if they had low E-cadherin protein (cell lines, as defined by their bi-modal index of 1.72) or a positive EMT gene signature score (patient tumors) (Figure 5A-5B) [6]. As expected, we observed a strong correlation between high E-cadherin levels and the miR-200 family members ( $\mathrm{r}=0.29-0.83$, corresponding to $\mathrm{P}<0.05-\mathrm{P}<0.001)$, which are established repressors of EMT (Figure 5A) [28]. We
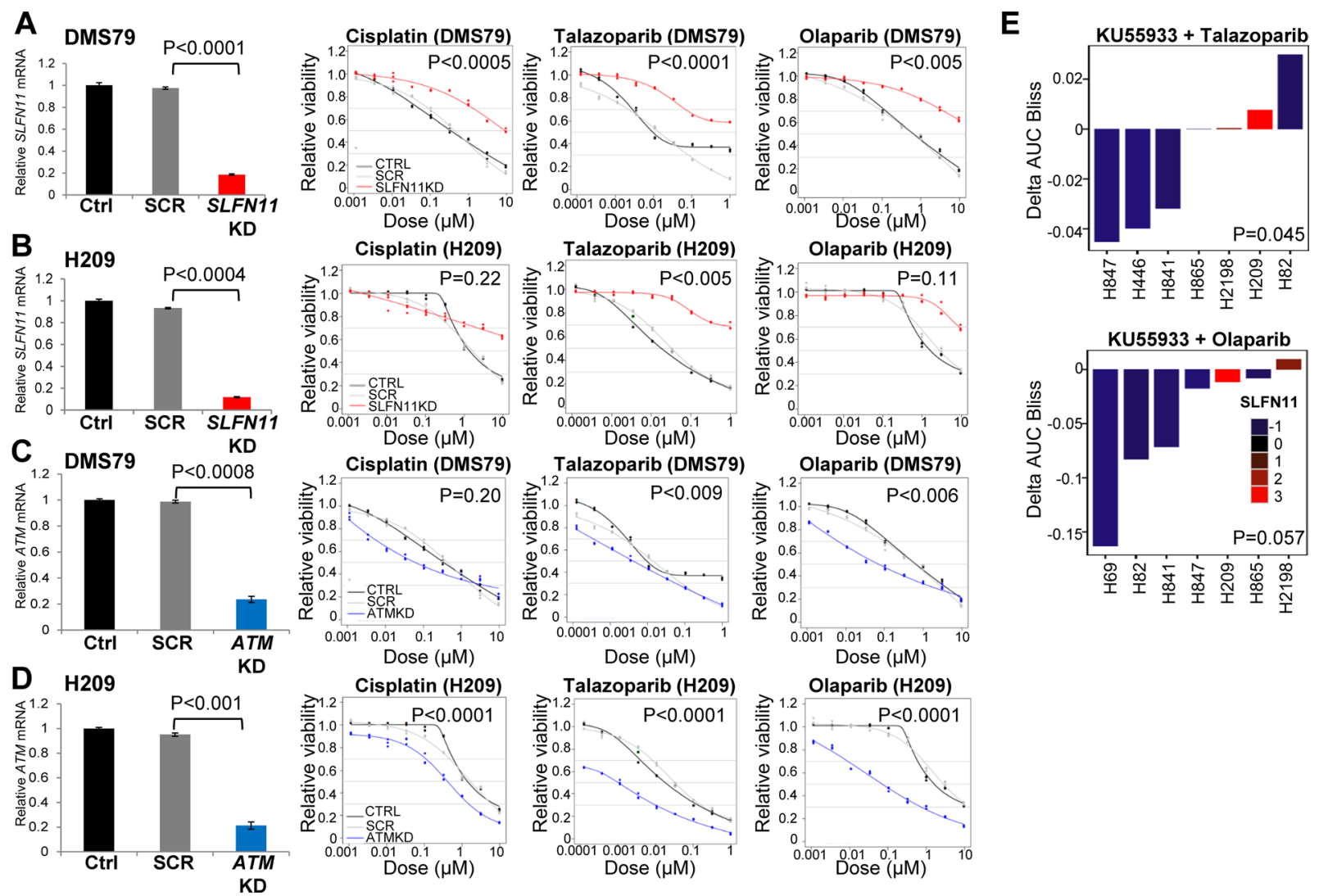

Figure 3: High SLFN11 and Low ATM Levels Maintain Sensitivity to PARP Inhibition and Chemotherapy. A, and B. Silencing of SLFN11 with siRNA in DMS79 and H209 SCLC cell lines (which have high SLFN11 expression levels, are sensitive to PARP inhibition, and have no ATM mutations), increases the cells' resistance to cisplatin, talazoparib and olaparib. C, and D. ATM knockdown increases sensitivity to cisplatin, talazoparib, and olaparib. siRNA effectively reduced SLFN11 and ATM. E. ATM inhibition sensitizes cell lines with low SLFN11 expression to PARP inhibition. The ATM inhibitor KU55933 plus talazoparib or olaparib was more effective in SCLC cell lines with lower SLFN11 levels. 
then evaluated cell lines and SCLC patient tumors for association between high E-cadherin levels or low EMT scores, respectively and a curated list of genes known to be markers of cancer stem cells [29, 30], involved in EMT [31], and associated with tumor progression in SCLC [32]. In both cell lines and tumors, we found that high E-cadherin is inversely correlated with the EMT gene signature score ( $\mathrm{rho}=-0.705, \mathrm{P}<0.001$ in cell lines, $\mathrm{rho}=$ $0.463, \mathrm{P}<0.001$ in tumors) (Figure 5A-5B). In general, epithelial cell lines and tumors were associated with cancer stem cell markers (CD24, ALDH1A1, and PROM1), markers of tumor aggressiveness (MYCL1 and ASCL1), and markers of apoptosis (BCL2 and BCL2L11). However, mesenchymal cell lines and tumors were primarily associated with EMT markers (ZEB1 and ZEB2). As SCLC is an extremely aggressive disease and $C D H 1$ levels are significantly higher in tumors than in normal adjacent tissues $(\mathrm{FC}=3.722 ; \mathrm{P}<0.0001)$, the correlation between $\mathrm{CDH} 1$ and these markers is not surprising (Supplementary Figure 7C).

Using an approach similar to that applied to SLFN11, we then investigated other drug classes for which E-cadherin might be a predictive marker of response in SCLC. Based on the bimodal index, we found that high E-cadherin level was associated with sensitivity to several drug types, including PARP inhibitors $(n=5)$, alkylating agents $(n=8)$, TOP2A/B inhibitors $(n=4)$, HDAC1 inhibitors $(n=3)$, BCL2 inhibitors $(n=3)$, and IGF1R inhibitors $(n=4)$ (Figure 5A5D). Many of these drug classes overlap with those to which SLFN11-expressing SCLC cell lines are sensitive, although E-cadherin and SLFN11 levels were not strongly correlated $(\mathrm{R}=0.233, \mathrm{P}=0.058$; Supplementary Figure 7B), suggesting that EMT and SLFN11 contribute independently to drug response.

\section{DISCUSSION}

Our findings suggest that high SLFN11 and low ATM expression levels predict PARP inhibitor response in PDX models and/or cell lines, whereas standard biomarkers of PARP inhibition defined in other cancer types (mutational burden, Myriad HRD score, and mutations in DDR genes) do not [33]. We also report for

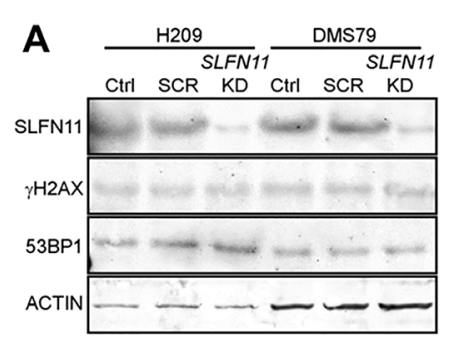

B
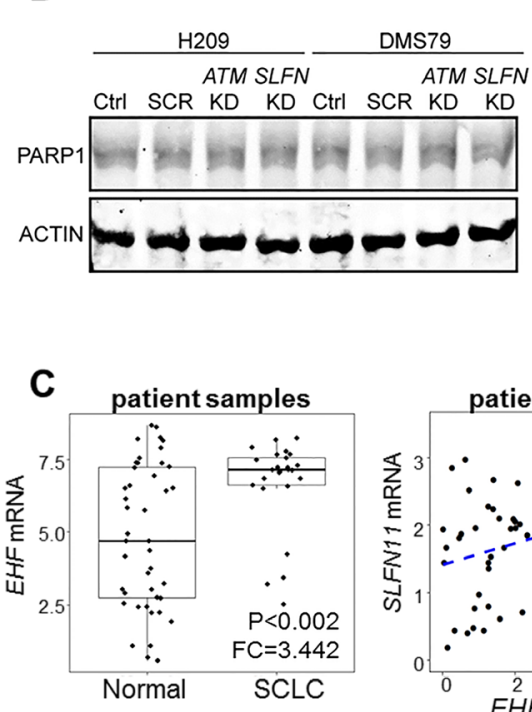
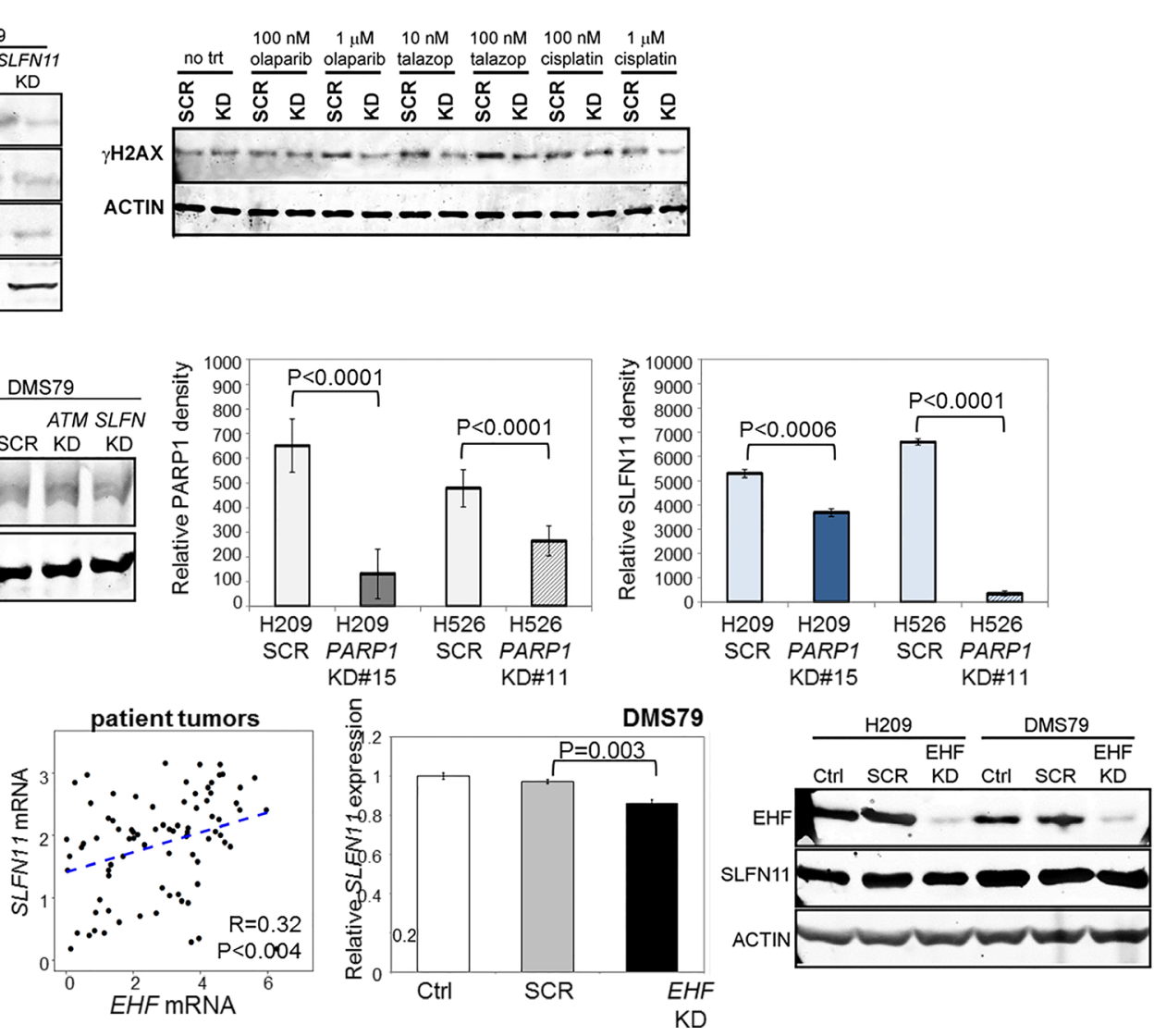

Figure 4: SLFN11 is Regulated by PARP1 and $\boldsymbol{E H F}$. A. Silencing of SLFN11 in H209 and DMS79 is insufficient to induce the expression of H2AX, a marker of DNA damage. Stimulation with olaparib, talazoparib, and cisplatin induced $\gamma \mathrm{H} 2 \mathrm{AX}$ in scrambled (SCR) but not SLFN11-knockdown cells. B. Silencing of SLFN11 or ATM does not affect PARP1 levels. Silencing of PARP1 in H209 and H526 cells reduces SLFN11 levels. C. EHF expression is elevated in SCLC tumors compared to normal adjacent tissues and is correlated with SLFN11 expression in tumors from treatment-naïve patients with early-stage SCLC. Silencing of EHF reduces SLFN11 mRNA and protein expression levels. 
the first time that EMT score and E-cadherin levels predict response to PARP inhibition, chemotherapy, and other targeted drugs in development for SCLC. Thus, multiple biomarkers, including SLFN11, ATM, and E-cadherin, may be important in predicting PARP inhibition or chemotherapy response in SCLC.

Although low ATM levels were associated with talazoparib response in PDX models, we had an insufficient number of ATM-low cell lines to test its predictive value across our cell line panel. However, we found that ATM knockdown increased PARP inhibitor and cisplatin sensitivity, suggesting that loss of ATM expression has a direct impact on drug sensitivity. Further supporting this, we found that the combination of ATM and PARP inhibitors overcame resistance to singleagent PARP inhibition in lines with low SLFN11. These findings - together with recent clinical data showing that $A T M$ mutations are associated with response to the
PARP inhibitor olaparib in prostate cancer patients [34]support the further investigation of ATM protein level as a candidate predictive biomarker. Moreover, inhibiting both ATM and PARP could help overcome drug resistance in patients whose tumors have low SLFN11 expression levels.

Based on levels of SLFN11 and ATM observed in treatment-naïve SCLC tumors, we expect that up to half of SCLC patients could respond to PARP inhibitors in the front-line setting. However, given that these cancers develop chemotherapy resistance within a few months after initial treatment, we predicted that SLFN11-a biomarker of cisplatin and PARP inhibitor responsemay be downregulated following platinum-based chemotherapy. Indeed, cell lines treated with cisplatin or PARP inhibitors had reduced SLFN11 levels. This implies that archival (diagnostic) tumor tissue may not accurately predict response to PARP inhibitors in patients who have

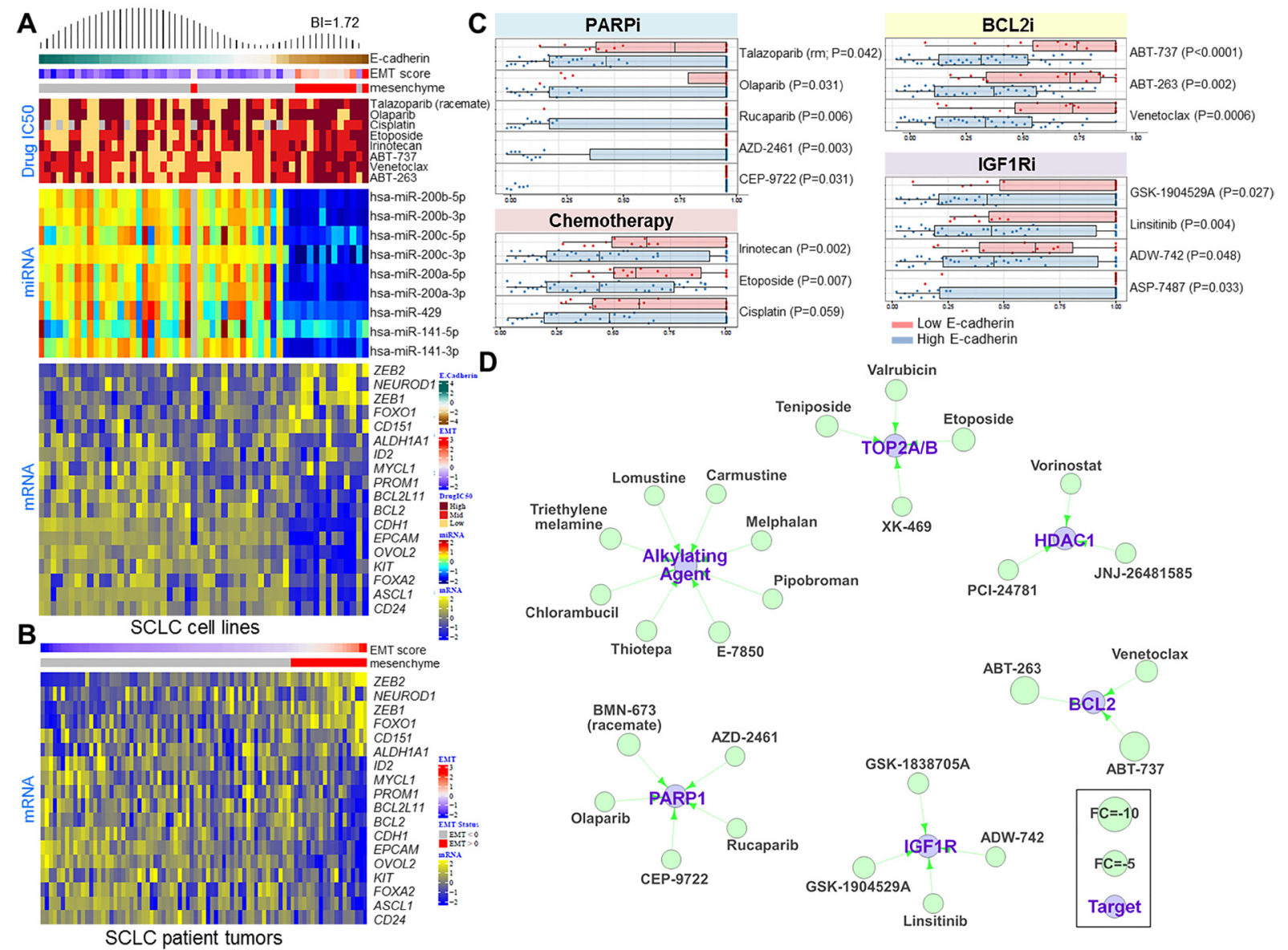

Figure 5: E-Cadherin Level Predicts Drug Sensitivity in SCLC. A. Heatmap of SCLC cell lines ranked by E-cadherin protein levels demonstrating a bimodal distribution pattern $(\mathrm{BI}=1.72)$. Cell lines with EMT scores greater than 0 are classified as mesenchymal. Correlation of E-cadherin expression and the $\mathrm{IC}_{50}$ values of PARP inhibitors, standard-of-care chemotherapy drugs, and BCL2 inhibitors. miR200 family microRNAs are strongly correlated with E-cadherin levels and EMT score. Expression analysis of a subset of genes involved in SCLC progression that are correlated with E-cadherin expression. B. A heatmap of 70 tumor samples from treatment-naïve SCLC patients ranked by EMT score shows the expression analysis of the same subset of genes as in the SCLC cell lines. C. High E-cadherin levels are associated with sensitivity to several drug classes, including PARP inhibitors, chemotherapy drugs, BCL2 inhibitors, and IGF1R inhibitors. D. A drug interaction network shows classes of drugs that are effective in SCLC cell lines with high E-cadherin levels. 
developed cisplatin resistance and, therefore, re-biopsy may be necessary to accurately assess biomarker status.

We found that SLFN11 directly regulated sensitivity to PARP inhibitors and cisplatin. Furthermore, loss of SLFN11 resulted in reduced DNA damage in response to cisplatin and PARP inhibitors, suggesting that its action is likely through regulation of DDR. Previous studies have shown that SLFN11 is recruited to sites of DNA damage, where it disrupts checkpoint maintenance and reduces HR repair [22]. We propose that high levels of SLFN11 may induce a type of synthetic lethality following PARP inhibitor or cisplatin treatment by negatively regulating DNA repair. Furthermore, based on our observation that silencing PARP1 reduced SLFN11 levels, loss of PARP1 expression (as described in some models of acquired PARP inhibitor resistance [24]), may mediate the loss of drug sensitivity directly through SLFN11 downregulation.

SLFN11 levels directly confer chemotherapy and/ or PARP inhibitor sensitivity in a number of cancer types, but it is unclear how this is controlled. Proposed mechanisms of SLFN11 regulation include IFN signaling [35], ETS transcription factor binding [25], and promoter methylation [27]. Several immune-related targets were found to be correlated with SLFN11 in early-stage SCLC, including 16 Type I IFN signaling pathway genes and targetable immune markers PDL1 and CTLA4. SLFN11 is expressed by T-cells and monocytes and is an IFNstimulated gene in peripheral blood mononuclear cells [36]. However, SLFN11 is not IFN-stimulated in SCLC cell lines suggesting that expression and regulation of SLFN11 by tumor and immune cells may be different. SLFN11 is also a potential surrogate marker of T-cell or macrophage infiltration and a biomarker for response to immunotherapy drugs targeting PDL1 and CTLA4, but this requires further testing in a clinical setting. Given the recent addition of immunotherapy to treatment options for relapsed SCLC (www.NCCN.org), these findings merit further investigation given their potential clinical impact [37]. The SLFN11 promoter contains several ETS binding sites and functions as an ETS factor response gene, with ETS transcription factors FLI1 and ETS1 having proposed roles in regulating SLFN11 expression in colon, breast, and prostate cancers [25]. In our analysis of ETS family members, only EHF is elevated in SCLC tumors, positively correlated with SLFN11 response (cell line and tumors) and predicts drug response. In contrast, $E H F$ is negatively correlated with SLFN11 expression in other cancers from NCI60 and CCLE data sets [25]. These findings imply that although SLFN11 functions as an ETS response gene, the particular ETS transcription factor that regulates $S L F N 11$ expression is cancer typespecific. In agreement with previous studies showing that hypermethylation regulates SLFN11 and is correlated with cisplatin sensitivity in NCI60 cell lines [27], we found two CpG regions near the SLFN11 TSS to be inversely correlated with SLFN11 mRNA levels and with in vitro sensitivity to both cisplatin and PARP inhibitors. However, treatment with decitabine and/or an HDAC inhibitor was insufficient to raise SLFN11 levels in SCLC cell lines. This suggests that methylation or other epigenetic modifications may not be dominant regulators of SLFN11 levels in SCLC, but may be useful as a biomarker of response to both cisplatin and PARP inhibitors.

We and others have previously shown an important role for EMT in modulating drug resistance in NSCLC. However, this is the first report linking EMT with resistance to multiple drug classes in SCLC. We found a significant association between EMT and resistance to PARP inhibitors, cisplatin, and other novel drug classes such as Bcl-2 inhibitors. In SCLC lines and patient tumors, loss of E-cadherin and high EMT gene signature score were associated with changes at the protein, miRNA, and mRNA level in genes and miRNA well known to regulate EMT, further supporting the presence of a mesenchymal subset of SCLC. This included downregulation of miR200 family members (important regulators of EMT, metastasis, and disease recurrence in NSCLC) [28, 38]; higher ZEB1/2, FOXO1, and NEUROD1; and decreased levels of stem cell markers (e.g., $A L D H 1 A 1)$ and other SCLC drivers (e.g., MYCL1, ASCL1) [32, 39]. ASCL1 and NEUROD1 are both necessary for SCLC tumor survival and disease progression, but regulate different downstream pathways, and whether they are involved in EMT is unknown [32].

To further explore the association between EMT and drug resistance, we expanded our analysis to a large screen of oncology drugs [12]. In addition to PARP inhibitors, this analysis revealed greater resistance of mesenchymal SCLC to alkylating agents, TOP2A/B inhibitors, BCL2 inhibitors, IGF1R inhibitors, and HDAC1 inhibitors. The higher response rate of E-cadherin high SCLC to BCL2 inhibitors (e.g., ABT-737, ABT-263, venetoclax) corresponded with higher BCL2 mRNA expression in the E-cadherin high cell lines and tumors, suggesting that BCL2 levels or EMT status could be used to help select patients for treatment with BCL-2 inhibitors and that pharmacologic strategies to reverse EMT may enhance BCL-2 inhibitor activity.

In conclusion, our findings support the notion that more than one biomarker is needed to predict SCLC response to PARP inhibition and/or chemotherapy and that expression of these biomarkers is dynamically regulated by drugs used in frontline therapy. In addition, our results show that SLFN11 and ATM directly regulate drug sensitivity, which suggests that therapeutically targeting these proteins may reduce or delay drug resistance in SCLC. Our results also show that the dependence of SLFN11 levels on PARP1 may represent a novel mechanism of acquired resistance to PARP inhibitors. Finally, E-cadherin levels and EMT score are linked with SCLC response to PARP inhibitors, chemotherapy, and 
other targeted therapies. Future studies should investigate methods to restore SLFN11 levels in resistant models and mechanisms regulating EMT, in order to develop means to therapeutically alter EMT while inhibiting PARP in SCLC.

\section{MATERIALS AND METHODS}

\section{Histology and IHC of PDX models}

A pathologist specializing in lung cancer (J.F.) reviewed each PDX model for hematoxylin and eosin and standard SCLC IHC markers (CD56, chromogranin A, and synaptophysin) to confirm that tumor pathology was consistent with SCLC.

IHC markers assessed in this study included those used routinely in the clinical diagnosis and characterization of lung cancers (synaptophysin, CD56, chromogranin A, Ki-67, and TTF1) and candidate biomarkers (SLFN11, ATM, and PARP).

\section{Mutation analysis}

DNA from PDX tumors was profiled using the clinical comprehensive genomic profiling FoundationOne hybrid-capture based NGS test (Foundation Medicine, Inc., Cambridge, MA) [40]. Mutation load was extrapolated to the exome or genome and estimated. The mutation load per megabase was calculated by dividing the total number of counted mutations by the coding region of the test (1.110 megabases). Foundation Medicine's SGZ algorithm was used to predict germline mutations [41]. Genomic instability was predicted for the PDX tumors and for 12 SCLC cell lines using the myChoice HRD assay (Myriad Genetics, Inc., Salt Lake City, UT).

\section{Cell lines}

Cells were grown in suggested media supplemented with fetal bovine serum and penicillin/streptomycin. Cells were passaged less than 6 months from the time they were received, and cell lines were routinely subjected to DNA fingerprinting as described previously [2].

\section{Gene knockdown}

For gene silencing, pooled small interfering RNAs (siRNAs) targeting SLFN11 (L-016764-01-0005) or ATM (L-003201-00-0005) and their corresponding scramble control (D-001810-10-05; GE Dharmacon) or EHF (S25399) and the Silencer Select Negative Control No. 2 (4390846, ThermoFisher Scientific) were transfected into H209 and DMS79 cells for 72 hours. The cells were then plated for 7-day proliferation assays or 24-h single-agent drug treatment. Knock down efficiency was validated by quantitative reverse transcription polymerase chain reaction and western blot analyses. PARPI was stably knocked down in $\mathrm{H} 209$ and $\mathrm{H} 526$ cell lines using plasmid vectors. The PARPI shRNAs were from GE Dharmacon
(V3LHS_391011, V3LHS_391013). Knock down efficiencies were assessed with western blotting.

\section{Drug treatments}

To determine effect of drug treatment on protein expression, H209 and H526 cells were plated for 24 $\mathrm{h}$ and then treated with $1 \mu \mathrm{M}$ cisplatin, $1 \mu \mathrm{M}$ olaparib, $100 \mathrm{nM}$ talazoparib. Cells were collected $72 \mathrm{~h}$ later, and protein lysates were isolated. SLFN11, E-cadherin, and ATM expression were assessed by western blotting. For the demethylation/HDAC inhibition experiments, H69, H526, H841, and H209 cells were plated for $24 \mathrm{~h}$ and then treated for $72 \mathrm{~h}$ with dimethyl sulfoxide or $5 \mu \mathrm{M}$ decitabine (Sigma Aldrich, St. Louis, MO) or with 100 $\mathrm{ng} / \mathrm{ml}$ trichostatin A (Sigma Aldrich) added in the last $16 \mathrm{~h}$. All experiments were performed in triplicate.

\section{ACKNOWLEDGMENTS}

We thank Evelyn Wang and Sanjay Chandriani for their help with mutational analysis and RNAseq, respectively, and Seema Mukherjee for generating PARPI knockdown cell lines.

This work was supported by: The NIH/NCI under award number R01CA207295 (LAB) P30CA016672 shRNA and ORFeome Core; University of Texas-Southwestern and MD Anderson Cancer Center Lung SPORE (5 P50 CA070907); through generous philanthropic contributions to The University of Texas MD Anderson Lung Cancer Moon Shot Program; MD Anderson Cancer Center Physician Scientist Award (LAB, DLG); Lee Clark Fellowship of The University of Texas MD Anderson Cancer Center, supported by the Jeane F. Shelby Scholarship Fund (LAB, DLG); an NCI Cancer Clinical Investigator Team Leadership Award (P30 CA016672) (LAB); The Sheikh Khalifa Bin Zayed Al Nahyan Institute for the Personalized Cancer Therapy's (IPCT's) Center for Professional Education and Training (LAB); the National Lung Cancer Research Partnership, made possible by the North Carolina Lung Cancer Partnership (LAB); the Lung Cancer Research Foundation (LAB); LUNGevity Foundation (LAB, DLG); and The Sidney Kimmel Foundation for Cancer Research (LAB); Rexanna's Foundation for Fighting Lung Cancer (DLG); The Stading Lung Cancer Research Fund (DLG). Abell Hangar Foundation Distinguished Professor Endowment (LAB).

\section{CONFLICTS OF INTEREST}

The authors declare no conflicts of interest.

\section{REFERENCES}

1. Byers LA, Rudin CM. Small cell lung cancer: Where do we go from here? Cancer. 2015; 121: 664-72. doi: 10.1002/ cncr. 29098. 
2. Byers LA, Wang J, Nilsson MB, Fujimoto J, Saintigny P, Yordy J, Giri U, Peyton M, Fan YH, Diao L, Masrorpour F, Shen L, Liu W, et al. Proteomic profiling identifies dysregulated pathways in small cell lung cancer and novel therapeutic targets including PARP1. Cancer Discov. 2012; 2: 798-811. doi: 10.1158/2159-8290.CD-12-0112.

3. Cardnell RJ, Feng Y, Diao L, Fan YH, Masrorpour F, Wang J, Shen Y, Mills GB, Minna JD, Heymach JV, Byers LA. Proteomic markers of DNA repair and PI3K pathway activation predict response to the PARP inhibitor BMN 673 in small cell lung cancer. Clin Cancer Res. 2013; 19: 63228. doi: 10.1158/1078-0432.CCR-13-1975.

4. McCabe N, Turner NC, Lord CJ, Kluzek K, Bialkowska A, Swift S, Giavara S, O'Connor MJ, Tutt AN, Zdzienicka MZ, Smith GC, Ashworth A. Deficiency in the repair of DNA damage by homologous recombination and sensitivity to poly(ADP-ribose) polymerase inhibition. Cancer Res. 2006; 66: 8109-15. doi: 10.1158/0008-5472. CAN-06-0140.

5. Murai J, Huang SY, Das BB, Renaud A, Zhang Y, Doroshow JH, Ji J, Takeda S, Pommier Y. Trapping of PARP1 and PARP2 by Clinical PARP Inhibitors. Cancer Res. 2012; 72: 5588-99. doi: 10.1158/0008-5472.CAN-12-2753.

6. George J, Lim JS, Jang SJ, Cun Y, Ozretic L, Kong G, Leenders F, Lu X, Fernandez-Cuesta L, Bosco G, Muller C, Dahmen I, Jahchan NS, et al. Comprehensive genomic profiles of small cell lung cancer. Nature. 2015; 524: 47-53. doi: 10.1038/nature14664.

7. Rudin CM, Durinck S, Stawiski EW, Poirier JT, Modrusan Z, Shames DS, Bergbower EA, Guan Y, Shin J, Guillory J, Rivers CS, Foo CK, Bhatt D, et al. Comprehensive genomic analysis identifies SOX2 as a frequently amplified gene in small-cell lung cancer. Nat Genet. 2012; 44: 1111-6. doi: 10.1038/ng.2405.

8. Peifer M, Fernandez-Cuesta L, Sos ML, George J, Seidel D, Kasper LH, Plenker D, Leenders F, Sun R, Zander T, Menon R, Koker M, Dahmen I, et al. Integrative genome analyses identify key somatic driver mutations of small-cell lung cancer. Nat Genet. 2012; 44: 1104-10. doi: 10.1038/ ng.2396.

9. Zoppoli G, Regairaz M, Leo E, Reinhold WC, Varma S, Ballestrero A, Doroshow JH, Pommier Y. Putative DNA/ RNA helicase Schlafen-11 (SLFN11) sensitizes cancer cells to DNA-damaging agents. Proc Natl Acad Sci U S A. 2012; 109: 15030-5. doi: 10.1073/pnas.1205943109.

10. Barretina J, Caponigro G, Stransky N, Venkatesan K, Margolin AA, Kim S, Wilson CJ, Lehar J, Kryukov GV, Sonkin D, Reddy A, Liu M, Murray L, et al. The Cancer Cell Line Encyclopedia enables predictive modelling of anticancer drug sensitivity. Nature. 2012; 483: 603-7. doi: 10.1038/nature11003.

11. Tian L, Song S, Liu X, Wang Y, Xu X, Hu Y, Xu J. Schlafen-11 sensitizes colorectal carcinoma cells to irinotecan. Anticancer Drugs. 2014; 25: 1175-81. doi: 10.1097/CAD.0000000000000151.
12. Polley E, Kunkel M, Evans D, Silvers T, Delosh R, Laudeman J, Ogle C, Reinhart R, Selby M, Connelly J, Harris E, Fer N, Sonkin D, et al. Small Cell Lung Cancer Screen of Oncology Drugs, Investigational Agents, and Gene and microRNA Expression. J Natl Cancer Inst. 2016; 108. doi: 10.1093/jnci/djw122.

13. Lok BH, Gardner EE, Schneeberger VE, Ni A, Desmeules P, Rekhtman N, de Stanchina E, Teicher BA, Riaz N, Powell SN, Poirier JT, Rudin CM. PARP Inhibitor Activity Correlates with SLFN11 Expression and Demonstrates Synergy with Temozolomide in Small Cell Lung Cancer. Clin Cancer Res. 2016. doi: 10.1158/1078-0432. CCR-16-1040.

14. Stewart CA, Tong P, Cardnell RJ, Sen T, Masrorpour F, Fan Y, Wang J, Byers LA. SLFN11 is a biomarker of sensitivity to PARP inhibition and chemotherapy in small cell lung cancer (SCLC). New Orleans: AACR; 2016.

15. Siolas D, Hannon GJ. Patient-derived tumor xenografts: transforming clinical samples into mouse models. Cancer Res. 2013; 73: 5315-9. doi: 10.1158/0008-5472. CAN-13-1069.

16. Anderson WC, Boyd MB, Aguilar J, Pickell B, Laysang A, Pysz MA, Bheddah S, Ramoth J, Slingerland BC, Dylla SJ, Rubio ER. Initiation and characterization of small cell lung cancer patient-derived xenografts from ultrasoundguided transbronchial needle aspirates. PLoS One. 2015; 10: e0125255. doi: 10.1371/journal.pone.0125255.

17. Mak MP, Tong P, Diao L, Cardnell RJ, Gibbons DL, William WN, Skoulidis F, Parra ER, Rodriguez-Canales J, Wistuba, II, Heymach JV, Weinstein JN, Coombes KR, et al. A Patient-Derived, Pan-Cancer EMT Signature Identifies Global Molecular Alterations and Immune Target Enrichment Following Epithelial-to-Mesenchymal Transition. Clin Cancer Res. 2016; 22: 609-20. doi: 10.1158/1078-0432.CCR-15-0876.

18. Cardnell RJ, Behrens C, Diao L, Fan Y, Tang X, Tong P, Minna JD, Mills GB, Heymach JV, Wistuba, II, Wang J, Byers LA. An Integrated Molecular Analysis of Lung Adenocarcinomas Identifies Potential Therapeutic Targets among TTF1-Negative Tumors, Including DNA Repair Proteins and Nrf2. Clin Cancer Res. 2015; 21: 3480-91. doi: 10.1158/1078-0432.CCR-14-3286.

19. Liu FW, Tewari KS. New Targeted Agents in Gynecologic Cancers: Synthetic Lethality, Homologous Recombination Deficiency, and PARP Inhibitors. Curr Treat Options Oncol. 2016; 17: 12. doi: 10.1007/s11864-015-0378-9.

20. Wang J, Wen S, Symmans WF, Pusztai L, Coombes KR. The bimodality index: a criterion for discovering and ranking bimodal signatures from cancer gene expression profiling data. Cancer Inform. 2009; 7: 199-216.

21. Schneider WM, Chevillotte MD, Rice CM. Interferonstimulated genes: a complex web of host defenses. Annu Rev Immunol. 2014; 32: 513-45. doi: 10.1146/ annurev-immunol-032713-120231. 
22. Mu Y, Lou J, Srivastava M, Zhao B, Feng XH, Liu T, Chen J, Huang J. SLFN11 inhibits checkpoint maintenance and homologous recombination repair. EMBO Rep. 2016; 17: 94-109. doi: 10.15252/embr.201540964.

23. Cremona CA, Behrens A. ATM signalling and cancer. Oncogene. 2014; 33: 3351-60. doi: 10.1038/onc.2013.275.

24. Helleday $T$. The underlying mechanism for the PARP and BRCA synthetic lethality: clearing up the misunderstandings. Mol Oncol. 2011; 5: 387-93. doi: 10.1016/j.molonc.2011.07.001.

25. Tang SW, Bilke S, Cao L, Murai J, Sousa FG, Yamade M, Rajapakse V, Varma S, Helman LJ, Khan J, Meltzer PS, Pommier Y. SLFN11 Is a Transcriptional Target of EWS-FLI1 and a Determinant of Drug Response in Ewing Sarcoma. Clin Cancer Res. 2015; 21: 4184-93. doi: 10.1158/1078-0432.CCR-14-2112.

26. Sato T, Kaneda A, Tsuji S, Isagawa T, Yamamoto S, Fujita T, Yamanaka R, Tanaka Y, Nukiwa T, Marquez VE, Ishikawa Y, Ichinose M, Aburatani H. PRC2 overexpression and PRC2-target gene repression relating to poorer prognosis in small cell lung cancer. Sci Rep. 2013; 3: 1911. doi: 10.1038/ srep01911.

27. Nogales V, Reinhold WC, Varma S, Martinez-Cardus A, Moutinho C, Moran S, Heyn H, Sebio A, Barnadas A, Pommier Y, Esteller M. Epigenetic inactivation of the putative DNA/RNA helicase SLFN11 in human cancer confers resistance to platinum drugs. Oncotarget. 2016; 7 : 3084-97. doi: 10.18632/oncotarget.6413.

28. Chen L, Gibbons DL, Goswami S, Cortez MA, Ahn YH, Byers LA, Zhang X, Yi X, Dwyer D, Lin W, Diao L, Wang J, Roybal JD, et al. Metastasis is regulated via microRNA-200/ZEB1 axis control of tumour cell PD-L1 expression and intratumoral immunosuppression. Nat Commun. 2014; 5: 5241. doi: 10.1038/ncomms6241.

29. Jahchan NS, Lim JS, Bola B, Morris K, Seitz G, Tran KQ, Xu L, Trapani F, Morrow CJ, Cristea S, Coles GL, Yang D, Vaka D, et al. Identification and Targeting of Long-Term Tumor-Propagating Cells in Small Cell Lung Cancer. Cell Rep. 2016; 16: 644-56. doi: 10.1016/j.celrep.2016.06.021.

30. Roudi R, Korourian A, Shariftabrizi A, Madjd Z. Differential Expression of Cancer Stem Cell Markers ALDH1 and CD133 in Various Lung Cancer Subtypes. Cancer Invest. 2015; 33: 294-302. doi: 10.3109/07357907.2015.1034869.

31. Park SM, Gaur AB, Lengyel E, Peter ME. The miR-200 family determines the epithelial phenotype of cancer cells by targeting the E-cadherin repressors ZEB1 and ZEB2. Genes Dev. 2008; 22: 894-907. doi: 10.1101/gad.1640608.

32. Borromeo MD, Savage TK, Kollipara RK, He M, Augustyn A, Osborne JK, Girard L, Minna JD, Gazdar AF, Cobb MH, Johnson JE. ASCL1 and NEUROD1 Reveal Heterogeneity in Pulmonary Neuroendocrine Tumors and Regulate Distinct Genetic Programs. Cell Rep. 2016; 16: 1259-72. doi: 10.1016/j.celrep.2016.06.081.
33. Lim D, Ngeow J. Evaluation of the methods to identify patients who may benefit from PARP inhibitor use. Endocr Relat Cancer. 2016; 23: R267-85. doi: 10.1530/ ERC-16-0116.

34. Mateo J, Carreira S, Sandhu S, Miranda S, Mossop H, Perez-Lopez R, Nava Rodrigues D, Robinson D, Omlin A, Tunariu N, Boysen G, Porta N, Flohr P, et al. DNARepair Defects and Olaparib in Metastatic Prostate Cancer. N Engl J Med. 2015; 373: 1697-708. doi: 10.1056/ NEJMoa1506859.

35. Puck A, Aigner R, Modak M, Cejka P, Blaas D, Stock1 J. Expression and regulation of Schlafen (SLFN) family members in primary human monocytes, monocyte-derived dendritic cells and T cells. Results Immunol. 2015; 5: 23-32. doi: 10.1016/j.rinim.2015.10.001.

36. Li M, Kao E, Gao X, Sandig H, Limmer K, Pavon-Eternod M, Jones TE, Landry S, Pan T, Weitzman MD, David M. Codon-usage-based inhibition of HIV protein synthesis by human schlafen 11. Nature. 2012; 491: 125-8. doi: 10.1038/ nature 11433 .

37. Antonia SJ, Lopez-Martin JA, Bendell J, Ott PA, Taylor M, Eder JP, Jager D, Pietanza MC, Le DT, de Braud F, Morse MA, Ascierto PA, Horn L, et al. Nivolumab alone and nivolumab plus ipilimumab in recurrent small-cell lung cancer (CheckMate 032): a multicentre, open-label, phase 1/2 trial. Lancet Oncol. 2016; 17: 883-95. doi: 10.1016/ S1470-2045(16)30098-5.

38. Patnaik SK, Kannisto E, Knudsen S, Yendamuri S. Evaluation of microRNA expression profiles that may predict recurrence of localized stage I non-small cell lung cancer after surgical resection. Cancer Res. 2010; 70: 36-45. doi: 10.1158/0008-5472.CAN-09-3153.

39. Huijbers IJ, Bin Ali R, Pritchard C, Cozijnsen M, Kwon MC, Proost N, Song JY, de Vries H, Badhai J, Sutherland K, Krimpenfort P, Michalak EM, Jonkers J, et al. Rapid target gene validation in complex cancer mouse models using re-derived embryonic stem cells. EMBO Mol Med. 2014; 6: 212-25. doi: 10.1002/emmm.201303297.

40. Frampton GM, Fichtenholtz A, Otto GA, Wang K, Downing SR, He J, Schnall-Levin M, White J, Sanford EM, An P, Sun J, Juhn F, Brennan K, et al. Development and validation of a clinical cancer genomic profiling test based on massively parallel DNA sequencing. Nat Biotechnol. 2013; 31: 102331. doi: 10.1038/nbt.2696.

41. Rosenberg JE, Hoffman-Censits J, Powles T, van der Heijden MS, Balar AV, Necchi A, Dawson N, O'Donnell PH, Balmanoukian A, Loriot Y, Srinivas S, Retz MM, Grivas $\mathrm{P}$, et al. Atezolizumab in patients with locally advanced and metastatic urothelial carcinoma who have progressed following treatment with platinum-based chemotherapy: a single-arm, multicentre, phase 2 trial. Lancet. 2016; 387: 1909-20. doi: 10.1016/S0140-6736(16)00561-4. 